PROCEEDINGS OF THE

AMERICAN MATHEMATICAL SOCIETY

Volume 130, Number 10, Pages 2845-2851

S 0002-9939(02)06408-0

Article electronically published on March 15, 2002

\title{
A PROOF OF WEINBERG'S CONJECTURE ON LATTICE-ORDERED MATRIX ALGEBRAS
}

\author{
JINGJING MA AND PIOTR J. WOJCIECHOWSKI
}

(Communicated by Lance W. Small)

Dedicated to Professor Melvin Henriksen on his 75th birthday

\begin{abstract}
Let $\mathbf{F}$ be a subfield of the field of real numbers and let $\mathbf{F}_{n}(n \geq 2)$ be the $n \times n$ matrix algebra over $\mathbf{F}$. It is shown that if $\mathbf{F}_{n}$ is a lattice-ordered algebra over $\mathbf{F}$ in which the identity matrix 1 is positive, then $\mathbf{F}_{n}$ is isomorphic to the lattice-ordered algebra $\mathbf{F}_{n}$ with the usual lattice order. In particular, Weinberg's conjecture is true.
\end{abstract}

Let $L$ be a totally ordered field, and let $L_{n}(n \geq 2)$ be the $n \times n$ matrix algebra over $L$. Then $L_{n}$ may be lattice-ordered by requiring that a matrix in $L_{n}$ is positive exactly when each of its entries is positive, that is, the positive cone is $\left(L^{+}\right)_{n}$. This lattice order is called the usual lattice order of $L_{n}$.

Let $\mathbf{Q}$ be the field of rational numbers. In 1966, Weinberg conjectured that $\left(\mathbf{Q}^{+}\right)_{n}$ is the only lattice order of $\mathbf{Q}_{n}$ (up to an isomorphism) such that $\mathbf{Q}_{n}$ is a lattice-ordered algebra ( $\ell$-algebra) over $\mathbf{Q}$ in which 1 is positive, and he proved his conjecture for $n=2$ [8]. Recently some conditions have been obtained to ensure an $\ell$-algebra $L_{n}$, in which 1 is positive, is isomorphic to the $\ell$-algebra $L_{n}$ with the usual lattice order [5], [7].

In this paper, we show that Weinberg's conjecture is true for a matrix $\ell$-algebra over any subfield of real numbers. More precisely, suppose that $\mathbf{F}$ is a subfield of the field of real numbers; it is shown that an $\ell$-algebra $\mathbf{F}_{n}$ over $\mathbf{F}$ in which 1 is positive is isomorphic to the $\ell$-algebra $\mathbf{F}_{n}$ with the usual lattice order.

We begin by collecting some definitions and results we will use later. The reader is referred to Birkhoff \& Pierce [2] and Fuchs [4] for the general theory of latticeordered rings ( $\ell$-rings). A partially ordered ring (po-ring) $R$ is an (associative) ring which is partially ordered, and in which i) $a \geq b$ implies $a+c \geq b+c$, for any $c \in R$, and ii) $a \geq 0$ and $b \geq 0$ imply $a b \geq 0$. Let $R$ be a po-ring. The set $R^{+}=\{a \in R: a \geq 0\}$ is called the positive cone of $R$. Clearly $R^{+}$is closed under addition and multiplication, and $R^{+} \cap-R^{+}=\{0\}$. Conversely, if $P$ is a subset of a ring $R$ which is closed under addition and multiplication, and satisfies $P \cap-P=\{0\}$, then the partial order $\geq$ defined by $a \geq b$ if and only if $a-b \in P$ makes $R$ into a po-ring with the positive cone equal to $P$. We will refer to such a

Received by the editors March 20, 2001 and, in revised form, May 16, 2001.

2000 Mathematics Subject Classification. Primary 06F25; Secondary 15A48.

Key words and phrases. Lattice-ordered algebra, matrix algebra.

The results in this paper were presented at the conference "Lattice-ordered groups and $f$-rings" at the University of Florida, March 2001. 
positive cone as a partial order of a po-ring $R$, and denote a po-ring $R$ with positive cone $P$ by $(R, P)$. If $R$ is a po-ring and an algebra over a totally ordered field $L$, then $R$ is called a partially ordered algebra (po-algebra) over $L$ if $L^{+} R^{+} \subseteq R^{+}$. A po-ring (po-algebra) is called an $\ell$-ring ( $\ell$-algebra) if the partial order is a lattice order. Similarly, partially ordered groups (po-groups) and lattice-ordered groups ( $\ell$ groups) and partially ordered vector spaces (po-vector spaces) and vector lattices over a totally ordered field can be defined. We skip these standard definitions, and refer the reader to [2] and [4].

An element $a>0$ in a po-group $G$ is called basic if the interval $[0, a]=\{b \in G$ : $0 \leq b \leq a\}$ is a chain. A subset $S$ of an $\ell$-group $G$ is called disjoint if $s>0$ for each $s \in S$ and $s \wedge t=0$ for any $s, t \in S, s \neq t$. If $G$ is an $\ell$-group and $a \in G$, then the absolute value of $a$ is $|a|=a \vee(-a)$, the positive part of $a$ is $a^{+}=a \vee 0$, and the negative part of $a$ is $a^{-}=(-a) \vee 0$.

For the rest of the paper, we always assume that $\mathbf{F}$ is a subfield of the totally ordered field $\mathbf{R}$ of real numbers; $\mathbf{F}_{n}$ is the $n \times n$ matrix algebra over $\mathbf{F}$, and $\mathbf{F}^{n}$ is the vector space $\mathbf{F} \oplus \ldots \oplus \mathbf{F}$ ( $n$ times) over $\mathbf{F}$. We also consider $\mathbf{F}^{n}$ as a topological subspace of the Euclidean space $\mathbf{R}^{n}$. If $\left(\mathbf{F}_{n}, P\right)$ is a po-algebra over $\mathbf{F}$ with the positive cone $P$, then we simply say that $P$ is a partial order of $\mathbf{F}_{n}$. The positive cone of a po-vector space $\mathbf{F}^{n}$ over $\mathbf{F}$ is simply called a cone in $\mathbf{F}^{n}$.

Let $P$ be a partial order of $\mathbf{F}_{n}$. A nonempty subset $S$ of $\mathbf{R}^{n}$ is said to be a $P$ invariant set if for every $f \in P, f S \subseteq S$. A cone $O$ in $\mathbf{F}^{n}$ is said to be a $P$-invariant cone in $\mathbf{F}^{n}$ if $O$ is also a $P$-invariant set. We remark that in these definitions, a $P$-invariant set is not necessarily a subset of $\mathbf{F}^{n}$, and although $P$-invariant cones can be defined more generally, we will only consider $P$-invariant cones in $\mathbf{F}^{n}$ in this paper. Obviously, $\{0\}$ is a $P$-invariant cone for every $P$. We will refer to this cone as the trivial $P$-invariant cone. Other cones will be called nontrivial.

Given a subset $K$ of $\mathbf{R}^{n}$, let

$$
\text { cone }_{\mathbf{F}}(K)=\left\{\sum \alpha_{i} v_{i}: \alpha_{i} \in \mathbf{F}^{+}, v_{i} \in K\right\} \text {. }
$$

It is clear that cone $\mathbf{F}(K) \subseteq \mathbf{F}^{n}$ exactly when $K \subseteq \mathbf{F}^{n}$. Also, it is easily verified that cone $\mathbf{F}(K)+\operatorname{cone}_{\mathbf{F}}(K) \subseteq \operatorname{cone}_{\mathbf{F}}(K)$ and $\mathbf{F}^{+} \operatorname{cone}_{\mathbf{F}}(K) \subseteq \operatorname{cone}_{\mathbf{F}}(K)$. But cone $_{\mathbf{F}}(K) \cap-$ cone $_{\mathbf{F}}(K) \neq\{0\}$ in general. In case $\mathbf{F}=\mathbf{R}$, many interesting properties of cones and matrices that leave a cone invariant are discussed in Berman \& Plemmons [1] and some papers referenced there. Note that if $K$ is finite, then cone $_{\mathbf{R}}(K)$ is closed in the Euclidean space $\mathbf{R}^{n}$ [1, Theorem 2.5] and if $K$ consists of $n$ linearly independent vectors in $\mathbf{R}^{n}$, then cone $\mathbf{R}(K)$ has a nonempty interior. It actually satisfies definition 2.10 of a proper cone in [1].

Theorem 1. Every nontrivial partial order $P$ of $\mathbf{F}_{n}$ has a nontrivial $P$-invariant cone in $\mathbf{F}^{n}$.

Proof. Consider the collection $\boldsymbol{M}$ of all the null spaces in $\mathbf{F}^{n}$ of the nonzero matrices from $P$. Let $N$ denote an element of $M$ with maximum dimension, and let $u \notin N$. Define $O=\{f u: f \in P$ and $f N=0\}$. The set $O$ is closed under addition and $\mathbf{F}^{+} O \subseteq O$. Also, if some $f u, g u \in O$ and $f u+g u=0$, then $(f+g) u=0$ and $(f+g) N=0$. Thus $(f+g)(\mathbf{F} u+N)=0$. But since $f+g \in P$ and $N \subset \mathbf{F} u+N$, it follows from the maximality of the dimension of $N$ that $f+g=0$. Therefore, $f=g=0$ and $f u=g u=0$. This proves that $O$ is a cone in $\mathbf{F}^{n}$. It is obvious that $O$ is nontrivial and $P$-invariant. 
Lemma 2. If $P$ is a directed partial order of $\mathbf{F}_{n}$ and $S \neq\{0\}$ is a $P$-invariant set, then $S$ contains $n$ linearly independent vectors over $\mathbf{R}$.

Proof. Let $M$ be the subspace of $\mathbf{R}^{n}$ generated by $S$. Then for every $f \in P$, $f M \subseteq M$. But then, since $P$ is directed, every matrix $g \in \mathbf{F}_{n}$ satisfies $g=$ $f_{1}-f_{2}$ with $f_{1}, f_{2} \in P$, so $g M=\left(f_{1}-f_{2}\right) M \subseteq M$, and hence $M$ is a nontrivial invariant subspace for every matrix in $\mathbf{F}_{n}$. Thus $M=\mathbf{R}^{n}$, so $S$ contains $n$ linearly independent vectors over $\mathbf{R}$.

Let $S$ be a subset of $\mathbf{R}^{n}$. The topological closure of $S$ in the Euclidean space $\mathbf{R}^{n}$ will be denoted by $\bar{S}$.

Lemma 3. If $P$ is a directed partial order of $\mathbf{F}_{n}$ and $O$ is a $P$-invariant cone in $\mathbf{F}^{n}$, then $\bar{O} \cap \overline{-O}=\{0\}$.

Proof. The set $\bar{O} \cap \overline{-O}$ is a $P$-invariant set. Moreover, since both $\bar{O}$ and $\overline{-O}$ are closed under addition and scalar multiplication by nonnegative real numbers, so is $\bar{O} \cap \overline{-O}$. If we suppose that $\bar{O} \cap \overline{-O} \neq\{0\}$, then by Lemma $2, \bar{O} \cap \overline{-O}$ contains a set $K$ of $n$ linearly independent vectors of $\mathbf{R}^{n}$ over $\mathbf{R}$. Therefore, cone $\mathbf{R}_{\mathbf{R}}(K) \subseteq \bar{O} \cap \overline{-O}$. Let $U$ be the interior of $\operatorname{cone}_{\mathbf{R}}(K)$. Note that $0 \notin U$. Since $U \cap-O \neq \emptyset, \bar{O} \cap-O \neq$ $\{0\}$, and hence by Lemma 2 , it contains a set $K_{1}$ of $n$ linearly independent vectors of $\mathbf{R}^{n}$ over $\mathbf{R}$. Let $U_{1}$ be the interior of $\operatorname{cone}_{\mathbf{R}}\left(K_{1}\right)$. Then $U_{1} \cap O \neq \emptyset$, since $U_{1} \subseteq$ cone $_{\mathbf{R}}\left(K_{1}\right) \subseteq \bar{O}$. Since $K_{1} \subseteq-O \subseteq \mathbf{F}^{n}, U_{1} \cap O \subseteq U_{1} \cap \mathbf{F}^{n} \subseteq$ cone $_{\mathbf{R}}\left(K_{1}\right) \cap \mathbf{F}^{n}$ $=$ cone $_{\mathbf{F}}\left(K_{1}\right) \subseteq-O$, and hence $U_{1} \cap O \subseteq O \cap-O$; so $O \cap-O \neq\{0\}$, which is a contradiction.

Note that we have just proven that if $P$ is a directed partial order of $\mathbf{F}_{n}$ and $O$ is a $P$-invariant cone in $\mathbf{F}^{n}$, then $\left(\mathbf{R}^{n}, \bar{O}\right)$ is a po-vector space over $\mathbf{R}$ with the positive cone $\bar{O}$, and $\bar{O}$ is $P$-invariant.

Lemma 4. Let $T$ be a subspace of $\mathbf{F}^{m}(m>1)$ over $\mathbf{F}$ which is totally ordered. If $\operatorname{dim}_{\mathbf{F}}(T)>1$, then $\overline{T^{+}} \cap \overline{-T^{+}} \neq\{0\}$.

Proof. First notice that $\bar{T}$ is a vector subspace of $\mathbf{R}^{m}$ over $\mathbf{R}$. Suppose that $\overline{T^{+}} \cap$ $\overline{-T^{+}}=\{0\}$. Since $T=T^{+} \cup-T^{+}$, we have that $\bar{T}=\overline{T^{+}} \cup \overline{-T^{+}}$. If we write $X=\bar{T} \backslash\{0\}, A=\overline{T^{+}} \backslash\{0\}=\bar{T} \backslash \overline{-T^{+}}$and $B=\overline{-T^{+}} \backslash\{0\}=\bar{T} \backslash \overline{T^{+}}$, then we have that $X=A \cup B$ and $A \cap B=\emptyset$, so $X$ is a union of two open, disjoint, nonempty subsets of $\bar{T}$, proving that $X$ is disconnected as a subset of $\bar{T}$. Since $\bar{T}$ is a Euclidean space, this is true only if $\operatorname{dim}_{\mathbf{R}}(\bar{T})=1$. Finally, since $T \subseteq \mathbf{F}^{m}$, we have $\operatorname{dim}_{\mathbf{F}}(T)=1$.

Corollary 5. Let $\mathbf{F}^{m}(m>1)$ be a po-vector space over $\mathbf{F}$ such that $\left(\mathbf{F}^{m}\right)^{+} \subseteq$ $\left(\mathbf{F}^{+}\right)^{m}$, and let $T \neq 0$ be a totally ordered subspace of $\mathbf{F}^{m}$. Then $T$ is onedimensional.

Proof. Since $T^{+} \subseteq\left(\mathbf{F}^{+}\right)^{m}, \overline{T^{+}} \subseteq \overline{\left(\mathbf{F}^{+}\right)^{m}}=\left(\mathbf{R}^{+}\right)^{m}$. Similarly, $\overline{-T^{+}} \subseteq\left(-\mathbf{R}^{+}\right)^{m}$. Since $\left(\mathbf{R}^{+}\right)^{m} \cap\left(-\mathbf{R}^{+}\right)^{m}=\{0\}, \overline{T^{+}} \cap \overline{-T^{+}}=\{0\}$, and hence $T$ is one-dimensional by Lemma 4.

A vector lattice over a totally ordered field $L$ is called Archimedean over $L$ if it has no nonzero bounded subspaces.

Corollary 6. Let $\left(\mathbf{F}_{n}, P\right)$ be an $\ell$-algebra over $\mathbf{F}$ with the positive cone $P$. If $P$ is contained in the usual lattice order $\left(\mathbf{F}^{+}\right)_{n}$, then $\left(\mathbf{F}_{n}, P\right)$ contains a set of exactly $n^{2}$ disjoint basic elements. 
Proof. By 2, Corollary 1, p.51], $\mathbf{F}_{n}$ is Archimedean over $\mathbf{F}$. So $\mathbf{F}_{n}\left(=\mathbf{F}^{n^{2}}\right.$ as a vector lattice over $\mathbf{F}$ ) is a vector lattice direct sum of finitely many totally ordered subspaces of $\mathbf{F}_{n}$ (see [3] p. 3.27] or [6] Theorem 2.12]). Each of these subspaces is one-dimensional by Corollary 5 Therefore, there exist $n^{2}$ disjoint basic elements.

Theorem 7. Let $\left(\mathbf{F}_{n}, P\right)$ be an $\ell$-algebra over $\mathbf{F}$. If for some vector $v \in \mathbf{F}^{n}$, $O=P v$ is a $P$-invariant cone in $\mathbf{F}^{n}$, then there exists a finite subset $K \subseteq \mathbf{F}^{n}$ such that $O=$ cone $_{\mathbf{F}}(K)$.

Proof. We know that $\mathbf{F}_{n}$ is a vector lattice direct sum of finitely many totally ordered subspaces, say, $\mathbf{F}_{n}=\bigoplus_{i=1}^{m} T_{i}$. For every $i, T_{i} v$ is a totally ordered subspace of $\left(\mathbf{F}^{n}, O\right)$ with the positive cone $\left(T_{i}\right)^{+} v$. Since $\overline{\left(T_{i} v\right)^{+}} \subseteq \bar{O}$ and $\overline{-\left(T_{i} v\right)^{+}} \subseteq \overline{-O}$, $\overline{\left(T_{i} v\right)^{+}} \cap \overline{-\left(T_{i} v\right)^{+}} \subseteq \bar{O} \cap \overline{-O}=\{0\}$ by Lemma 3. So by Lemma 4, $\operatorname{dim}_{\mathbf{F}}\left(T_{i} v\right) \leq 1$. This means that for every $i=1, \ldots, m$, there exists $f_{i} \in T_{i}^{+}$such that $T_{i} v=\mathbf{F} f_{i} v$. Therefore $O=P v=\left(\sum_{i=1}^{m} T_{i}^{+}\right) v=\sum_{i=1}^{m}\left(T_{i}^{+}\right) v=\sum_{i=1}^{m}\left(T_{i} v\right)^{+}=\sum_{i=1}^{m} \mathbf{F}^{+} f_{i} v=$ cone $_{\mathbf{F}}(K)$, where $K=\left\{f_{i} v: i=1, \ldots, m\right\}$.

Let $O$ be a nontrivial cone in $\mathbf{F}^{n}$. If $O=\operatorname{cone}_{\mathbf{F}}(K)$ for some finite subset $K$ of $\mathbf{F}^{n}$, then $O$ will be called a polyhedral cone. If there exists such a $K$ which has precisely $n$ elements, and they are linearly independent, then $O$ will be called $a$ simplicial cone. In the case $\mathbf{F}=\mathbf{R}$, these notions coincide with definitions 2.4 and 2.13 from [1. If $K$ is a minimal finite set with the property that $O=\operatorname{cone}_{\mathbf{F}}(K)$ for a polyhedral cone $O$, then the vectors from $K$ will be called the edges of $O$. Let $P$ be a directed partial order of $\mathbf{F}_{n}$, and $O$ a nontrivial $P$-invariant cone in $\mathbf{F}^{n}$. Then, by Lemma 2, $O$ contains $n$ linearly independent vectors; therefore, if $O=$ cone $_{\mathbf{F}}(K)$ for some $K \subseteq \mathbf{F}^{n}$, then $K$ contains at least $n$ elements.

Theorem 8. Let $P$ be a lattice order of $\mathbf{F}_{n}$. Then every nontrivial P-invariant cone in $\mathbf{F}^{n}$ contains a minimal nontrivial P-invariant cone in $\mathbf{F}^{n}$. In particular, there exists a minimal nontrivial P-invariant cone.

Proof. First, we consider a chain $\left\{P v_{\alpha}\right\}$ of nontrivial $P$-invariant cones $P v_{\alpha}$ and show that $\bigcap P v_{\alpha}$ is a nontrivial $P$-invariant cone. Let $S$ denote the unit sphere in $\mathbf{R}^{n}$. The family $\left\{\overline{P v_{\alpha}} \cap S\right\}$ is a chain of closed nonempty sets in the space $S$, and therefore their intersection $\bigcap_{\alpha}\left(\overline{P v_{\alpha}} \cap S\right) \neq \emptyset$ since $S$ is compact. Therefore, $\bigcap_{\alpha} \overline{P v_{\alpha}} \neq\{0\}$. Since this set is $P$-invariant, it contains a subset $K$ of $n$ linearly independent vectors from $\mathbf{R}^{n}$ over $\mathbf{R}$ by Lemma 2, By Theorem[7, for each $\alpha, P v_{\alpha}=$ cone $_{\mathbf{F}}\left(K_{\alpha}\right)$ for some finite subset $K_{\alpha} \subseteq \mathbf{F}^{n}$. It is clear that $\overline{P v_{\alpha}}=\overline{\text { cone }_{\mathbf{F}}\left(K_{\alpha}\right)}=$ cone $_{\mathbf{R}}\left(K_{\alpha}\right)$ and $\overline{P v_{\alpha}} \cap \mathbf{F}^{n}=$ cone $_{\mathbf{R}}\left(K_{\alpha}\right) \cap \mathbf{F}^{n}=$ cone $_{\mathbf{F}}\left(K_{\alpha}\right)$, where cone $\mathbf{R}\left(K_{\alpha}\right) \cap$ $\mathbf{F}^{n}=$ cone $_{\mathbf{F}}\left(K_{\alpha}\right)$ since a polyhedral cone containing $n$ linearly independent vectors is a union of simplicial cones and the equality is clearly true for a simplicial cone. Thus cone $\mathbf{R}_{\mathbf{R}}(K) \cap \mathbf{F}^{n} \subseteq \overline{P v_{\alpha}} \cap \mathbf{F}^{n}=$ cone $_{\mathbf{F}}\left(K_{\alpha}\right)=P v_{\alpha}$, for every $P v_{\alpha}$, and hence cone $_{\mathbf{R}}(K) \cap \mathbf{F}^{n} \subseteq \cap P v_{\alpha}$, so $\cap P v_{\alpha} \neq\{0\}$.

Now let $O$ be a nontrivial $P$-invariant cone in $\mathbf{F}^{n}$. Let $0 \neq v \in O$. Then $P v$ is contained in $O$, so $P v$ is a nontrivial $P$-invariant cone. By the above argument and Zorn's Lemma, $P v$ contains a minimal nontrivial $P$-invariant cone, so $O$ contains a minimal nontrivial $P$-invariant cone. Finally, because of Theorem 1 there exists a minimal nontrivial $P$-invariant cone in $\mathbf{F}^{n}$. 
Lemma 9. Let $O$ be a polyhedral cone in $\mathbf{F}^{n}$, and let $k$ be an edge of $O$. If $0<$ $u<k$ for some $u \in \mathbf{F}^{n}$, then $u=\alpha k$ for some $\alpha \in \mathbf{F}^{+}$. In particular, the edges of $O$ are basic elements of the partial order $O$ in $\mathbf{F}^{n}$.

Proof. Let $K=\left\{k, k_{1}, \ldots, k_{m}\right\}$ be minimal with the property $O=\operatorname{cone}_{\mathbf{F}}(K)$. Then, $u=\alpha k+\sum_{j=1}^{m} \alpha_{j} k_{j}$ and $k-u=\beta k+\sum_{j=1}^{m} \beta_{j} k_{j}$, where $\alpha, \beta, \alpha_{j}, \beta_{j} \in \mathbf{F}^{+}$ for $j=1, \ldots, m$. Therefore, $k=(\alpha+\beta) k+\sum_{j=1}^{m}\left(\alpha_{j}+\beta_{j}\right) k_{j}$. Since $K$ is minimal, we must have $\alpha+\beta=1$, and $\alpha_{j}=\beta_{j}=0$ for $j=1, \ldots, m$. So $u=\alpha k$.

Theorem 10. Let $\left(\mathbf{F}_{n}, P\right)$ be an $\ell$-algebra over $\mathbf{F}$ and let $O$ be a minimal nontrivial $P$-invariant cone in $\mathbf{F}^{n}$. Then:

(1) $O$ is a lattice order in $\mathbf{F}^{n}$.

(2) $O$ is a simplicial cone in $\mathbf{F}^{n}$, so $O$ has exactly $n$ disjoint edges.

Proof. (1) By Lemma 2, $O$ contains $n$ linearly independent vectors $v_{i}, i=1, \ldots, n$, over $\mathbf{F}$. Let $v=\sum_{i} v_{i}$. Then $P v=O$ from the minimality of $O$, and hence $O$ is a polyhedral cone by Theorem 7 , Let $k$ be an edge of $O$. There exists a matrix $f_{k} \in P$ such that $k=f_{k} v=\sum f_{k} v_{i}$. Since $k$ is an edge of $O$, by Lemma 9, we have that for all $i=1, \ldots, n, f_{k} v_{i}=\alpha_{i} k$ for some scalars $\alpha_{i} \geq 0$. Consequently, $\operatorname{rank}\left(f_{k}\right)=1$. Thus the null space $N$ of $f_{k}$ has dimension $n-1$. By the proof of Theorem 1 $O^{\prime}=\{f v: f \in P$ and $f N=0\}$ is a nontrivial $P$-invariant cone. Obviously, $O^{\prime} \subseteq P v$ and we conclude that $O=O^{\prime}=P v$.

Since $O$ contains $n$ linearly independent vectors, it is a directed partial order in $\mathbf{F}^{n}$. Let $u \in \mathbf{F}^{n}$. There exist $u_{1}, u_{2} \in O$ such that $u=u_{1}-u_{2}$. Let $u_{1}=f_{1} v$ and $u_{2}=f_{2} v$ for some $f_{1}, f_{2} \in P$ and $f_{1} N=f_{2} N=0$. Then $u=\left(f_{1}-f_{2}\right) v$. Let $f=f_{1}-f_{2}$. Then $f^{+} v \in O$ since $f^{+} \in P$. Similarly $\left(f^{+}-f\right) v=f^{-} v \in O$. Thus $f^{+} v$ is an upper bound for both 0 and $u$. Below we show that $f^{+} v$ is the least upper bound of 0 and $u$.

Let $0, u \leq w$. Then $w \in O$, so $w=g v$ for some $g \in P$ with $g N=0$. Similarly $w-u \in O$ implies $w-u=h v$ for some $h \in P$ with $h N=0$. Thus $w-u=$ $g v-f v=h v$, so $(g-f) v=h v$ and also $(g-f) N=h N=0$. Therefore, $g-f=h$ since $v \notin N$ and $N$ has dimension $n-1$. Since $h \in P$, we have $g=h+f \geq f$, and since $g \in P$, we obtain $g \geq f^{+}$. Thus $g-f^{+} \in P$, and hence $\left(g-f^{+}\right) v \in O$; so $g v-f^{+} v \geq 0$; i.e., $w \geq f^{+} v$.

(2) By (1) $O$ is a lattice order of $\mathbf{F}^{n}$. By Lemma 9, the edges of $O$ are basic elements in this lattice order, and therefore they are linearly independent over $\mathbf{F}$. Therefore there are at most $n$ disjoint edges, and thus $O$ has exactly $n$ disjoint edges.

Theorem 11. Every $\ell$-algebra $\left(\mathbf{F}_{n}, P_{1}\right)$ is isomorphic to an $\ell$-algebra $\left(\mathbf{F}_{n}, P_{2}\right)$ with $P_{2} \subseteq\left(\mathbf{F}^{+}\right)_{n}$, and $\left(\mathbf{F}^{+}\right)^{n}$ is a minimal $P_{2}$-invariant cone.

Proof. Let $O_{1}$ be a minimal nontrivial $P_{1}$-invariant cone in $\mathbf{F}^{n}$. By Theorem 10. $O_{1}$ is simplicial. Let $k_{1}, \ldots, k_{n}$ be the edges of $O_{1}$. Let $\psi$ be the $n \times n$ matrix with the columns $k_{1}, \ldots, k_{n}$. Since the edges form a linearly independent set, $\psi$ is nonsingular and yields both an algebra isomorphism $\mathbf{F}_{n} \rightarrow \psi^{-1} \mathbf{F}_{n} \psi$ and a vector space isomorphism $\mathbf{F}^{n} \rightarrow \psi^{-1} \mathbf{F}^{n}$. Let $P_{2}=\psi^{-1} P_{1} \psi$. Then $\left(\mathbf{F}_{n}, P_{2}\right)$ becomes an $\ell$-algebra which is isomorphic to $\left(\mathbf{F}_{n}, P_{1}\right)$. If we let $O_{2}=\psi^{-1} O_{1}$, then $O_{2}=$ $\psi^{-1}\left(\mathbf{F}^{+} k_{1}+\ldots+\mathbf{F}^{+} k_{n}\right)=\psi^{-1} \psi\left(\mathbf{F}^{+}\right)^{n}=\left(\mathbf{F}^{+}\right)^{n}$, and it is clear that $O_{2}$ is a minimal $P_{2}$-invariant cone. Finally, $P_{2} \subseteq\left(\mathbf{F}^{+}\right)_{n}$ since $\left(\mathbf{F}^{+}\right)^{n}$ is $P_{2}$-invariant. 
Given $f \in \mathbf{F}_{n}$, the transpose of $f$ is denoted by $f^{T}$. It is well-known that if $P$ is a partial order (lattice order) of $F_{n}$, then so is $P^{T}=\left\{f^{T}: f \in P\right\}$.

Theorem 12. Let $\left(\mathbf{F}_{n}, P\right)$ be an $\ell$-algebra over $\mathbf{F}$ such that $1 \in P$. Then $\left(\mathbf{F}_{n}, P\right)$ is isomorphic to the $\ell$-algebra $\left(\mathbf{F}_{n},\left(\mathbf{F}^{+}\right)_{n}\right)$.

Proof. By Theorem 11 we may assume that $P \subseteq\left(\mathbf{F}^{+}\right)_{n}$ and $O=\left(\mathbf{F}^{+}\right)^{n}$ is a minimal nontrivial $P$-invariant cone. We show that $P=\left(\mathbf{F}^{+}\right)_{n}$. Let $v \in O$ be any vector with all components strictly positive. Then $P v \neq\{0\}$, and since $O$ is minimal, $P v=O$. Hence there are $n$ matrices $f_{i} \in P$ such that $f_{i} v=e_{i}, i=1, \ldots, n$, where $e_{i}$ denotes the vector in which the $i^{\text {th }}$ component is 1 and the other components are zero. Since every $f_{i}, i=1, \ldots, n$, has all its entries nonnegative, all rows of $f_{i}$ except the $i^{\text {th }}$ one are zero rows. It follows that $f_{i}^{T} e_{j}=0$ for $i \neq j$, and $f_{i}^{T} v \neq 0, i, j=$ $1, \ldots, n$. Let $N_{i}$ denote the subspace of $\mathbf{F}^{n}$ spanned by $\left\{e_{1}, \ldots, e_{i-1}, e_{i+1}, \ldots, e_{n}\right\}$. Then for every $i=1, \ldots, n, f_{i}^{T} N_{i}=0$. Now $\left(\mathbf{F}_{n}, P^{T}\right)$ is an $\ell$-algebra over $\mathbf{F}$. It follows from the proof of Theorem 1 that the sets $O_{i}=\left\{f^{T} v: f^{T} N_{i}=0, f \in P\right\}$, $i=1, \ldots, n$, are nontrivial $P^{T}$-invariant cones. By Lemma 2 each $O_{i}$ contains $n$ linearly independent vectors. For each $1 \leq i \leq n$, suppose $f_{i j}^{T} v, j=1, \ldots, n$, are linearly independent vectors in $O_{i}$, where $f_{i j} \in P$ and $f_{i j}^{T} N_{i}=0$. Then it is clear that for each $i, f_{i j}, j=1, \ldots, n$, are linearly independent over $\mathbf{F}$, and all rows of $f_{i j}$ except the $i^{\text {th }}$ one are zero rows. Then it follows that the whole set $\left\{f_{i j}: 1 \leq i, j \leq n\right\}$ is linearly independent over $\mathbf{F}$. Since $P \subseteq\left(\mathbf{F}^{+}\right)_{n}$, by Corollary [6] $\left(\mathbf{F}_{n}, \bar{P}\right)$ contains a set $G$ of $n^{2}$ disjoint basic elements. Then $P=\sum_{g \in G} \mathbf{F}^{+} g$. Since $\left\{f_{i j}: 1 \leq i, j \leq n\right\} \subseteq P$, each $f_{i j}$ is a nonnegative linear combination of elements in $G$. Since $\left\{f_{i j}: 1 \leq i, j \leq n\right\}$ is linearly independent, each $g \in G$ must appear in at least one such linear combination with a nonzero coefficient from $\mathbf{F}^{+}$. Keeping in mind that all entries of the matrices $g \in G$ are nonnegative numbers, we conclude that each $g \in G$ has all but one row zero.

Now let $G=\left\{g_{i j}: 1 \leq i, j \leq n\right\}$. Then for every pair of indices $i, j=1, \ldots, n$, there exists a matrix from $G$, say, $g_{i j}$, whose $i j^{\text {th }}$ entry is not 0 . Moreover, since $1 \in P$, for each $i=1, \ldots, n$ there is a matrix from $G$ which we will, without loss of generality, call $g_{i i}$, such that it has only a nonzero $i i^{\text {th }}$ entry. Let $e_{i j}$, $i, j=1, \ldots, n$, denote the usual matrix units in $\mathbf{F}_{n}$. Then $g_{i i}=\lambda_{i i} e_{i i}$ for some $0<\lambda_{i i} \in \mathbf{F}$, for every $i=1, \ldots, n$. Therefore, for every $i=1, \ldots, n$, the matrices $e_{i i} \in P$. Now for every $i, j=1, \ldots, n, g_{i j} e_{j j}=\lambda_{i j} e_{i j}$ for some $0<\lambda_{i j} \in \mathbf{F}$. Thus $\left\{e_{i j}: i, j=1, \ldots, n\right\} \subseteq P$, and hence $P=\left(\mathbf{F}^{+}\right)_{n}$.

\section{ACKNOWLEDGMENTS}

The authors are very grateful to Professors A. M. W. Glass, Melvin Henriksen, Charles Holland, Stephen McCleary, and Stuart Steinberg for valuable discussions and comments during the preparation of this paper.

\section{REFERENCES}

1. A. Berman and R. J. Plemmons, Nonnegative Matrices in the Mathematical Sciences, Academic Press, 1979. MR 82b:15013

2. G. Birkhoff and R. S. Pierce, Lattice-ordered rings, An. Acad. Brasil. Cienc. 28 (1956), 41-69. MR 18:191d

3. P. Conrad, Lattice-ordered groups, Tulane Lecture Notes, Tulane University, 1970.

4. L. Fuchs, Partially ordered algebraic systems, Akademia Kiado, Budapest. MR 30:2090

5. J. Ma, Lattice-ordered matrix algebras with the usual lattice order, J. of Algebra 228 (2000), 406-416. MR 2001d:16066 
6. S. A. Steinberg, Finitely-valued f-modules, Pacific J. Math. 40 (1972), 723-737. MR 46:5205

7. S. A. Steinberg, On the scarcity of lattice-ordered matrix algebras II, Proc. Amer. Math. Soc. 128 (2000), no. 6, 1605-1612. MR 2000j:06011

8. E. C. Weinberg, On the scarcity of lattice-ordered matrix rings, Pacific J. Math. 19 (1966), 561-571. MR 34:2635

Department of Mathematical Sciences, University of Houston-Clear lake, 2700 Bay Area Boulevard, Houston, Texas 77058

E-mail address: ma@cl.uh.edu

Department of Mathematical Sciences, The University of Texas at El Paso, El Paso, TEXAs 79968

E-mail address: piotr@math.utep.edu 